\title{
Clamping effects on mechanical stability and energy dissipation in nanoresonators based on carbon nanotubes
}

\section{Journal Article}

Author(s):

Kumar, Lalit; Jenni, Laura V.; Haluska, Miroslav; Roman, Cosmin; Hierold, Christofer

Publication date:

2019-11-14

Permanent link:

https://doi.org/10.3929/ethz-b-000381402

Rights / license:

In Copyright - Non-Commercial Use Permitted

Originally published in:

Journal of Applied Physics 126(18), https://doi.org/10.1063/1.5099862

Funding acknowledgement:

153292 - Q-factor enhancement in resonating CNTs (SNF) 


\section{Clamping Effects on Mechanical Stability and Energy Dissipation in Nanoresonators based on Carbon Nanotubes}

Lalit Kumar, a) Laura V. Jenni, Miroslav Haluska, Cosmin Roman, and Christofer Hierold

Micro- and Nanosystems, Department of Mechanical and Process Engineering, ETH Zurich, Tannenstrasse 3, 8092 Zurich, Switzerland

(Dated: 21 October 2019)

With continuous downscaling of resonators, clamping is expected to significantly impact the mechanical stability as well as the energy dissipation mechanisms, especially at the nanoscale. For understanding of clamping effects at nanoscale, we here report an experimental investigation of a same nanotube based resonator subjected to two different clamping configurations. We investigate clamping associated stability and damping mechanisms by pushing the resonator into the non-linear regime. The nanotube was first dry-transferred and suspended between source-drain palladium electrodes resulting in a bottom clamped configuration. A selective top metallization process by platinum atomic layer deposition applied later resulted in a top-bottom clamped configuration. Large nanotube motional amplitude leading to a non-linear Duffing response initiated small slippage of the nanotube. This instability in clamping was seen in both clamping configurations and was measured as an irreversible resonance frequency downshift. For the measured resonator devices, gate induced nanotube tension in the range of $58-71 \mathrm{pN}$ was estimated to overcome clamping forces and initiate slipping. In terms of energy dissipation, top metallization process was accompanied by a reduction in amplitude dependent non-linear damping and Q-factor enhancement. Subjecting the same nanotube to both clamping configurations allowed for a direct comparison of clamping and quantification of non-linear damping. In the present case, non-linear damping was observed at an estimated nanotube motional amplitude of $11 \mathrm{~nm}$ (and higher), being dominant in bottom clamped configuration suggesting the origin of this non-linear damping to partially stem from external mechanisms, in addition to other possible internal dissipation path reported such as viscoelastic effects.

Keywords: NEMS, carbon nanotube, clamping, non-linear damping, resonators

\section{INTRODUCTION}

Resonant sensors based on nanoelectromechanical systems (NEMS) have pushed the limits of detection in applications such as room temperature mass sensing down to attograms ${ }^{1}$ with a potential to measure atomic masses $^{2}$. Such advancements were possible due to the ultra-small mass of the resonators such as a carbon nanotube resonating at high frequencies. However with increased resonance frequencies, as a result of miniaturization, energy dissipation (captured by the inverse Q-factor - $Q^{-1}$ ) has shown an increasing trend ${ }^{3}$ thereby countering the advantages of downscaling and limiting the sensing performance. Low Q-factors observed in nanoresonators have been attributed to various fundamental intrinsic dissipation mechanisms such as phonon-scattering $Q_{P S}^{-14}$, thermoelastic damping $Q_{T E D}^{-1}{ }^{5}$, surface losses $Q_{S L}^{-16}$ as well as extrinsic dissipation mechanisms, which includes clamping losses $Q_{C L}^{-17}$ and fluid damping $Q_{F D}^{-18}$. The overall energy dissipation of the resonators obtained from the summation of all mechanisms is represented by the effective quality factor $\mathrm{Q}$ given $\mathrm{by}^{3}$

$$
\frac{1}{Q}=\frac{1}{Q_{C L}}+\frac{1}{Q_{T E D}}+\frac{1}{Q_{S L}}+\frac{1}{Q_{P S}}+\frac{1}{Q_{F D}}
$$

For nanoresonators, limitations of low $\mathrm{Q}$ factors have been overcome by decreasing the temperature to millikelvin ranges ${ }^{9}$ and thereby decreasing phononassisted and thermoelastic damping ${ }^{10}\left(Q_{P S}^{-1}, Q_{T E D}^{-1}\right)$. Surface treatments ${ }^{11}$ and defect engineering ${ }^{12}$ have been shown to enhance Q-factors by reducing surface losses $\left(Q_{S L}^{-1}\right)$. Furthermore, operation under low pressure conditions makes fluid damping $Q_{F D}^{-1}$ negligible ${ }^{13}$. Clamping losses $\left(Q_{C L}^{-1}\right)$ due to anchor or contact electrodes have been argued to be a dominant loss mechanism as the resonator scales down to subnanometers ${ }^{14,15}$. Theoretical work in understanding clamping losses has been reported through analytical models $^{16,17}$ and molecular simulations. ${ }^{14,18}$ Yet, experimental observations, in particular low Q-factors at room temperature, are largely unexplained with respect to theoretical expectations. Most of the reported theoretical and experimental work on nanoresonators including carbon nanotubes have assumed perfect clamping conditions. However, recent reported results have experimentally proven the finite and weak adhesion forces leading to clamping instability in CNT based nanoresonators making a case against the assumption of perfect clamping conditions ${ }^{19,20}$. Very few experimental observations of dissipation due to clamping ${ }^{21,22}$ have been reported, which limits the general understanding of clamping effects on nanoresonators, and motivates the following research. 

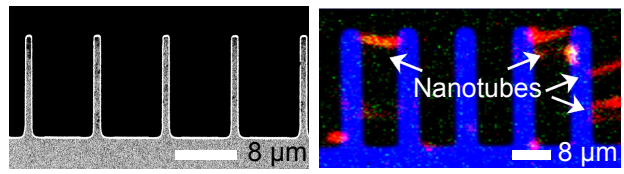

(a)
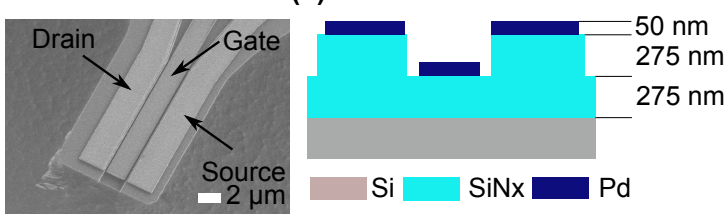

(b)

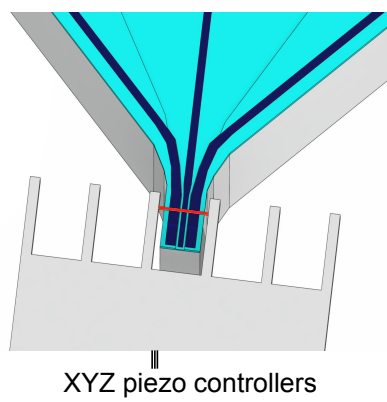

(c)

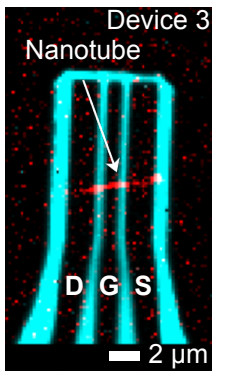

(d)

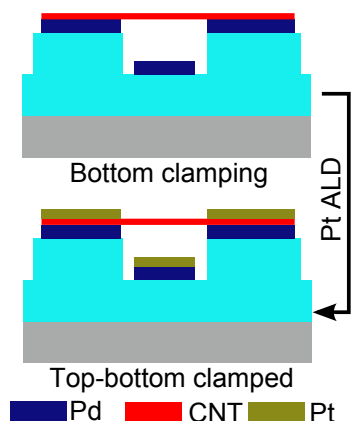

(e)

FIG. 1. (a) Scanning electron microscope (SEM) image (left) of fork-like structure used as growth substrate for carbon nanotubes. After CNT-growth, Raman spectroscopy was used to localize the nanotubes (right) (b) SEM image (left) of a fabricated device with a FET architecture (right) without CNT. (c) The shortlisted nanotube was manually dry-transferred from the growth substrate to the FET device using a micro-manipulator. (d) Raman image of a CNT FET based resonator device after dry transfer of nanotube. (e) Schematic of two different clamping configurations-bottom clamped obtained after dry-transfer and top-bottom clamped obtained from selective platinum metallization onto the electrodes.

\section{FABRICATION AND METHODS}

The resonators used in this investigation are based on a carbon nanotube. Our carbon nanotube resonator devices are fabricated on silicon substrates with a field-effect-transistor (FET) architecture as shown in Figure $1 \mathrm{~b}$ with a designed channel length of $2 \mu \mathrm{m}$ and an approximate channel-gate distance of $0.27 \mu \mathrm{m}$. Firstly, an insulating layer of $550 \mathrm{~nm}$ silicon nitride was deposited through plasma enhanced chemical vapor deposition (PECVD). An approximate $0.27 \mu \mathrm{m}$ trench depth for gate electrode was etched through reactive ion etching (RIE) without etch stop layer. The FET electrodes - source(S), drain(D) and gate(G) were fabricated by palladium evaporation in a lift-off process with chromium as an adhesion layer. Finally, to make these devices dry-transfer compatible, the nitride and approximately $25 \mu \mathrm{m}$ of the underlying silicon were etched by inductively coupled plasma RIE (ICP-RIE) ${ }^{23}$. The assembly of carbon nanotubes onto FET devices was done through a mechanical, ultra-clean dry transfer of $\mathrm{CNTs}^{24}$. The CNTs were grown through chemical vapor deposition (CVD) at $850^{\circ} \mathrm{C}$ on designated fork shaped chips fabricated on silicon-on-insulator (SOI) wafers as shown in Figure 1a. They were characterized by non-destructive Raman spectroscopy for localization and structural quality assessment (Figure 1a). Nanotubes with non-detectable $\mathrm{D}$ peak (indicative of the presence of structural defects) were selected and then drytransferred $^{24}$ onto FET devices.

The dry-transferred nanotube is attached onto the palladium source-drain electrodes resulting in a bottomclamped configuration. To investigate the effect of additional top clamping, a $20 \mathrm{~nm}$ platinum layer was selectively deposited ${ }^{23,25}$ by atomic layer deposition (ALD) onto the palladium electrodes resulting in a top-bottom clamped configuration as shown in Figure 1e. The selective deposition was performed at $300^{\circ} \mathrm{C}$ with alternating pulses of $\mathrm{MeC}_{P} \mathrm{PtMe}_{3}$ and oxygen. The oxygen pressure controls the nucleation delay on non-catalyzing surfaces. Thus, during the ALD process, platinum is expected to deposit only on the catalyzing palladium electrodes. The process, an elaborate description of which can be found in [23], was performed with an oxygen pressure of 7.5 mtorr with 500 cycles resulting in an approximate platinum layer of $20 \mathrm{~nm}$. In contrast to conventional bottom clamped nanotube resonators ${ }^{1,9,19,26}$ or top-clamped nanotube resonators ${ }^{13}$ (with metal on the top side of the nanotubes only. The nanotubes are as-grown directly on silicon device substrates with $\mathrm{Si} / \mathrm{SiO}_{2}$ on the bottom side of the nanotube), top-bottom clamping configuration in nanotube resonators have not been reported to our knowledge. This selective top metallization allows us to study two different clamping conditions and also enables a direct comparison of clamping in case of a same nanotube resonator. In contrast to experimental investigations reported by Aykol et al. ${ }^{19}$ - focusing on clamping and mechanical stability in CNT beam resonators and Rieger et $a ._{.}^{22}$ - focusing on clamping induced energy dissipation in silicon nitride cantilevers, this research addresses both mechanical stability and energy dissipation using the same material (CNT) in a beam resonator configuration. In the present case, three devices have been investigated as summarized (additional information in supplementary informationsection 2) in Table I. Device 1 with top-bottom clamping and Device 2 with bottom clamping were fabricated from two different nanotubes. To further separate clamping effects from nanotube parameter variations, Device 3 was subjected to both - bottom clamping and top-bottom clamping configuration. Raman spectroscopy, DC transport measurement and RF resonance characterization (supplementary informationsection $1,2,3$ ) on the two clamping configurations were performed for detailed experimental investigation of the 
TABLE I. Measured resonator devices

\begin{tabular}{lcc}
\hline \hline & Top-bottom clamped & Bottom clamped \\
\hline Different Nanotube & Device 1 & Device 2 \\
Same Nanotube & Device 3 & Device 3 \\
\hline \hline
\end{tabular}

clamping effects. All electrical measurements were performed at a pressure of $10^{-4}$ mbar. A resistive heater and temperature sensor mounted onto the sample stage were used in a feedback loop to maintain a constant temperature of $298 \mathrm{~K}$ during the measurement cycles.

\section{CLAMPING STABILITY}

Under the hypothesis of weak clamping conditions between the nanotube and metal electrodes ${ }^{19}$ and to further study and quantify clamping strength, we drive our resonators into the non-linear regime at high DC gate biases. The resonators then exhibit a typical Duffing response as shown in Figure 2a,b. As a result, an irreversible resonance frequency downshift was observed as shown in Figure 2. This was attributed to the high CNT motional amplitude which leads to small slippage of the CNT over the underlying palladium electrode. This allows for a decrease in the internal tension of the nanotube and is observed as a resonance frequency downshift. A detailed experimental investigation of reduction in the internal tension in bottom clamped nanotube devices has been reported in our previous work ${ }^{20}$.

The weak clamping behavior and resonance frequency downshift curves for both clamping configurations is shown in Figure 2c. For top-bottom clamped configuration (Device 1), clamping instability was observed as a resonance frequency decrease from 65.7 $\mathrm{MHz}$ and saturating to $55 \mathrm{MHz}$ (plotted for a DC gate bias of $2 \mathrm{~V}$ ). A similar down-shift was also observed with bottom clamped devices (Device 2 and Device 3 plotted for a DC gate bias of $1.5 \mathrm{~V}$ ) until saturation was reached. Clamping strength, presumably arising from van-der-Waals forces between carbon and metal atoms i.e. $\mathrm{Pd}-\mathrm{C}$ interactions for bottom clamping and $\mathrm{Pd}-\mathrm{C}-$ $\mathrm{Pt}$ interactions for top-bottom clamping, may vary for each nanotube-electrode clamping configuration resulting in variations in the required DC gate bias to induce clamping instability. Considering the model for the first flexural beam mode with resonance frequency ${ }^{19}$

$f_{0}^{T}=\sqrt{\left(\frac{1}{2 L} \sqrt{\frac{T_{\text {clamping }}+T_{\text {gate }}}{\rho A}}\right)^{2}+\left(\frac{1}{2 \pi} \frac{4.73^{2}}{L^{2}} \sqrt{\frac{E I}{\rho A}}\right)^{2}}$

where E, A, L, $\rho$, I are nanotube parameters defined as the Young's modulus, cross-sectional area, suspended length, volumetric mass density and moment of inertia respectively. $T_{\text {gate }}$ is the nanotube tension due to

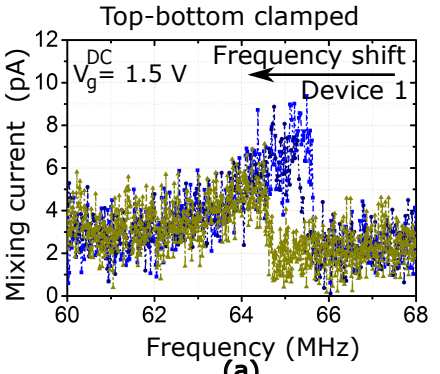

(a)
Bottom clamped

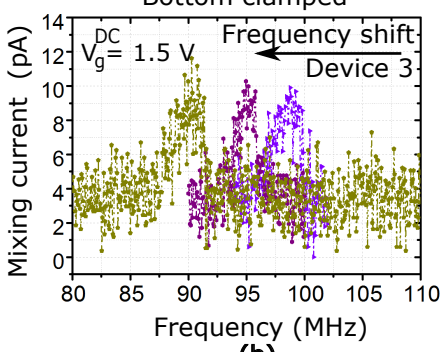

(b)

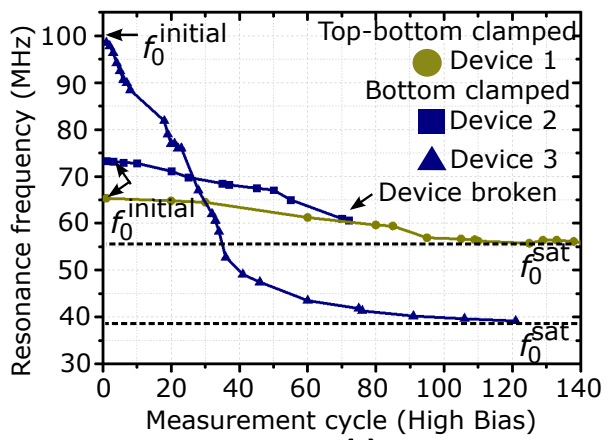

(c)

FIG. 2. (a) Decrease in the resonance frequency for top bottom clamped device 1 driven at DC gate bias of $V_{g}^{D C}=$ $1.5 V\left(V_{g}^{a c}=15 \mathrm{mV}, V_{d s}^{a c}=10 \mathrm{mV}\right.$ and $\left.V_{d s}^{D C}=10 \mathrm{mV}\right)$; (b) Decrease in resonance frequency for bottom clamped device 3 driven at $\mathrm{DC}$ gate bias of $V_{g}^{D C}=1.5 \mathrm{~V}\left(V_{g}^{a c}=\right.$ $18 m V, V_{d s}^{a c}=10 \mathrm{mV}$ and $V_{d s}^{D C}=10 \mathrm{mV}$ );(c) Clamping instability observed in top-bottom clamped (Device 1) and bottom clamped devices (Device 2, Device 3) as an irreversible resonance frequency downshift; plotted at a DC gate bias of 2 $\mathrm{V}$ and $1.5 \mathrm{~V}$ respectively. One measurement cycle is defined as five frequency sweeps performed to obtain a resonance spectral response $(\mathrm{b}, \mathrm{c})$. The $\mathrm{X}$ axis is approximated to fit all three devices in the same overview. The large CNT motional amplitude initiates small nanotube slippage and hence lowers the resonance frequency.

electrostatic DC gate bias and $T_{\text {clamping }}$ is the nanotube tension taking into account the clamping effect ${ }^{19}$. The first term under the square root corresponds to the string mode (to account for nanotube tension) and the second term corresponds to the beam mode (for zero tension). In comparison to numerical simulations for a beam under tension, the frequency-tension model (Eq. 2) can be used for nanotube tension estimation with an uncertainty of $6 \%$. With increasing DC gate bias, the nanotube is subjected to two simultaneous effects - Increasing $T_{\text {gate }}$ observed as an increase in the resonance frequency and secondly, decreasing $T_{\text {clamping }}$ due to slippage and mechanical stress relaxation ${ }^{20}$ which is observed as a decrease in resonance frequency. To quantify threshold for weak clamping and clamping forces, we keep the AC gate bias low $V_{g}^{a c}=3 m V$ and track the resonance frequencies when subjected to continuously varying DC gate bias as shown in Figure 3a.

Figure 3a shows the resonance frequencies subjected to two cycles of increasing DC gate biases on a bottom 


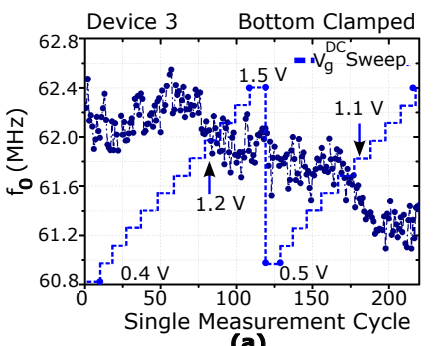

(a)
Device $3 \quad$ Bottom Clamped

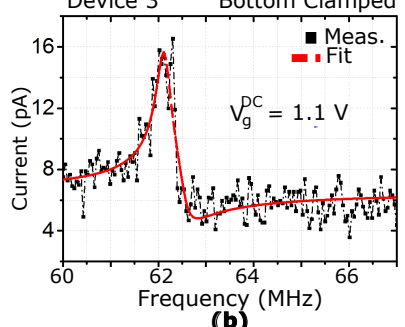

(b)

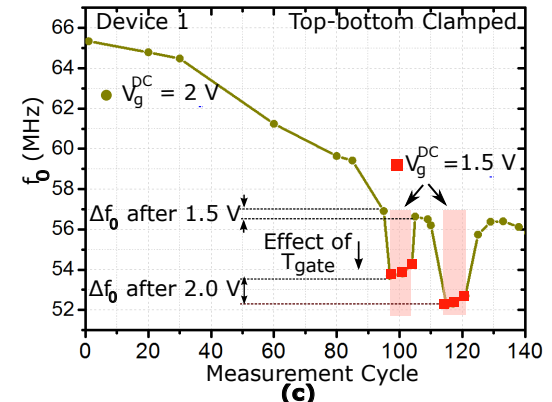

(c)

FIG. 3. (a) History of the resonance frequency for bottom clamped device 3 measured with increasing steps of $V_{g}^{D C}$ from 0.4 to $1.5 \mathrm{~V}$. Each single measurement cycle (shown in (b)) was performed ten times for each $V_{g}^{D C}$. Resonance frequency downshift due to weak clamping behavior was observed between $1.1 \mathrm{~V}<V_{g}^{D C}<1.2 \mathrm{~V}$ as indicated by the arrow. The spectral responses were measured with $\left(V_{g}^{a c}=3 \mathrm{mV}, V_{d s}^{a c}=10 \mathrm{mV}\right.$ and $\left.V_{d s}^{D C}=10 \mathrm{mV}\right)$; (c) Resonance frequency downshift for top-bottom clamped Device 1 subjected to DC gate bias of $1.5 \mathrm{~V}$ (shaded area, red) and $2 \mathrm{~V}$ (green). The sudden drop/rise in resonance frequency is attributed to DC bias dependent nanotube tension $T_{\text {gate }}{ }^{-}$ Eq. 2. Relative frequency downshift is used as a comparison for clamping instability. A higher electrostatic drive $(2 \mathrm{~V})$ leads to enhanced instability in clamping resulting in a larger resonance frequency downshift $\Delta f_{0}$ in comparison to $1.5 \mathrm{~V}$ bias.

clamped device. It should be mentioned here that with increasing DC gate bias, resonance frequency would be subjected to an interplay of additional simultaneous effects such as DC gate bias induced nanotube tension, spring hardening and possible drift and frequency fluctuations. However, the effect of resonance frequency downshift trend under large enough actuation forces is clearly visible for all three devices as shown in Figure 2c (additional measurements in supplementary informationsection 7 ). With the focus on $f_{0}$ decrease for slipping effect, a resonance frequency downshift (pointed by arrows) was observed between $1.1 \mathrm{~V}$ and $1.2 \mathrm{~V}$ suggesting the onset of slipping. We extract the minimum CNT motional amplitude by considering the spectral response obtained at $V_{g}^{D C}=1.1 \mathrm{~V}$ shown in Figure $3 \mathrm{~b}$, to be equal to $\sim 7.4 \mathrm{~nm}$ (supplementary information-section 4). This would correspond to $\sim 70 \mathrm{pN} \pm 14 \mathrm{pN}$ of gate induced tension (supplementary information-section 4) required to overcome clamping forces and initiate slipping. A higher DC gate bias is likely to enhance this weak clamping behavior. The effect of two different DC gate biases is shown in Figure 3c on a top-bottom clamped device 1 , where a frequency downshift $\Delta f_{0}$ of 0.13 $\mathrm{MHz}$ and $1.43 \mathrm{MHz}$ was observed with $1.5 \mathrm{~V}$ and 2 V DC gate bias respectively, suggesting an increased instability in clamping with higher DC electrostatic drive. From all three devices using $\mathrm{E}=1 \mathrm{TPa}$, a range for $T_{\text {gate }}=58-71 \mathrm{pN}$ was estimated to overcome clamping forces. The first effect mentioned previously of increasing $V_{g}^{D C}$ (and $T_{\text {gate }}$ ) and increasing $f_{0}$ and vice versa is more significantly visible in device 1 as compared to device 3 . Continuous measurements were performed with various DC gate biases until the saturation of resonance frequencies was observed (further information about the history of the measurements performed on devices until saturation can be found in supplementary informationsection 3). Saturation of resonance frequencies after repeated measurements allows us to experimentally quantify the residual tension due to clamping effects $T_{\text {clamping }}$ for a desired nanotube-clamping configuration. In case of zero tension, the first theoretical fundamental resonance frequency (beam mode) for the fabricated trench width of $2 \mu \mathrm{m}$ (L) and radius 1 to $1.5 \mathrm{~nm}$ would be $21 \pm 4 \mathrm{MHz}$. This estimation considers the shortest possible suspended nanotube length over the trench for our fabricated FET devices with a Young's modulus $\mathrm{E}=1 \mathrm{TPa}$ assuming a defect free nanotube and hence represents an upper bound to the extracted resonance frequency. The presence of residual tension in the nanotube allows for the observed resonance frequency saturation at $f_{0}^{s a t}=55 \mathrm{MHz}$ in top-bottom clamped Device 1 and $f_{0}^{s a t}=40 \mathrm{MHz}$ in bottom clamped Device 3 . Variations in surface roughness, frictional forces, van-derWaals force interactions and nanotube parameters would influence the residual tension leading to differences in $f_{0}^{\text {initial }}$ and $f_{0}^{\text {sat }}$. For the present case, using Eq. 2 with $f_{0}^{T}=f_{0}^{s a t}$, we estimate $T_{\text {clamping }}=150 \pm 35 \mathrm{pN}$ for Device 1, and $T_{\text {clamping }}=47 \pm 11 p N$ for Device 3 (supplementary information-section 4). These numbers represent a lower bound estimation (by using upper limits in the fundamental beam mode).

\section{CLAMPING AND ENERGY DISSIPATION}

While driving the resonator into the non-linear regime gave insight into clamping strength and stability, energy dissipation due to clamping, has not been discussed so far. In the framework of non-linear dynamics, we account for energy dissipation in our resonator devices by introducing non-linear terms ${ }^{27}$

$$
m \frac{d^{2} z}{d t^{2}}+b \frac{d z}{d t}+\eta z^{2} \frac{d z}{d t}+k z+\alpha z^{3}=F_{\text {drive }} \cos (\omega t)
$$

where $m, b, k$ relate to mass, linear damping and spring constant for the fundamental resonance mode and $\eta$ and $\alpha$ model the non-linear damping and non-linear spring respectively. The cubic term $\alpha z^{3}$ corresponds to 
geometric non-linearities and is observed as a non-linear Duffing response at higher gate bias as shown in Figure $2 a, b$. The total energy dissipation for such a system is contributed by both $b(d z / d t)$ and $\eta z^{2}(d z / d t)$ with the latter becoming dominant at large amplitude motion $\left(z^{2}\right.$ dependency). Due to variations in tension and nanotube parameters, damping parameters $(b, \eta)$ might also vary from device to device.

To verify the effect of clamping and exclude variations in nanotube parameters, Device 3, an already saturated (at $40 \mathrm{MHz}$ ) bottom clamped device, was subjected to a selective platinum ALD process resulting in a topbottom clamped configuration. Two different clamping configurations on the same nanotube device allows us for a direct comparison of clamping effects. A direction comparison of Q-factors reveal up to two folds improvement due to top-metallization in topbottom clamped device was shown in Figure 4. The measurements were repeated and the extracted Q-factors were plotted for various DC gate biases as shown in Figure $4 \mathrm{c}^{28}$. Taking into account the Q-factor variations, visible $\mathrm{Q}$-factor dependency on $V_{g}^{D C}$ was observed for bottom clamped device. The Q-factors showed a decreasing trend with increasing DC gate bias in bottom clamping configuration. In contract to top-bottom clamping, Q-factors remains similar (within the error bars). Such a behavior in bottom clamped devices was attributed to non-linear energy dissipation being dependent on nanotube motional amplitude. Experimental observations of non-linear amplitude dependent broadening have been previously reported on carbon nanotube and graphene resonators ${ }^{26}$. For linear damping with a damping coefficient $b$, the resonance width $\left(\Delta f=f_{0} / Q=b / 2 \pi m\right)$ is independent of the driving force. For the non-linear damping case, the resonance width broadens with increasing driving force $F_{\text {drive }}$. We increase the driving force as a function of $V_{g}^{a c}$ but keep it low enough to prevent the onset of Duffing non-linearity. The effect of non-linear damping in the form of spectral broadening is visible strongly for bottom clamped configuration as compared to topbottom clamping as shown in Figure 4d.

For bottom clamped configuration, the dominant effect of $\eta z^{2}(d z / d t)$ is visible for increasing drive force. In the limit, when $b(d z / d t)$ is ignored, the expected resonance width at a given resonance frequency $f_{0}$ is given by ${ }^{26}$

$$
\Delta f=0.032 \frac{\eta^{1 / 3} F_{d r i v e}^{2 / 3}}{m f_{0}^{2 / 3}}
$$

The experimental data fits in good agreement with Eq. 4 resulting in an extracted parameter $\eta=$ $160 \mathrm{~kg} \mathrm{~m} \mathrm{~m}^{-2} \mathrm{~s}^{-1}$. For top-bottom clamped nanotube, the dominant effect of $b(d z / d t)$ is visible. Using $\Delta f=$ $b / 2 \pi m\left(\Delta f \propto F^{0}\right.$ in Figure $\left.4 \mathrm{~d}\right)$, we extract $b=3.24 \times$ $10^{-15} \mathrm{~kg} \mathrm{~s}^{-1}$ for top-bottom clamped device.

Analysis for non-linear damping was also performed for other bottom clamped device 2 and top-clamped device

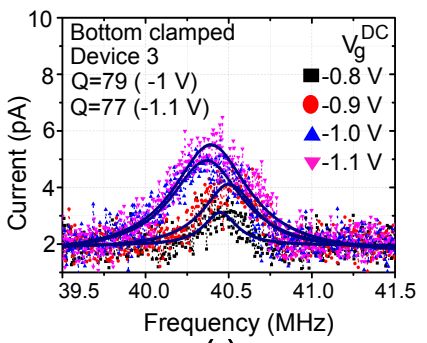

(a)

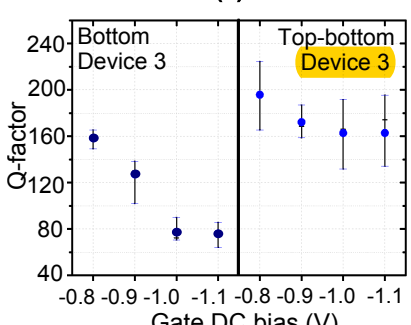

(c)

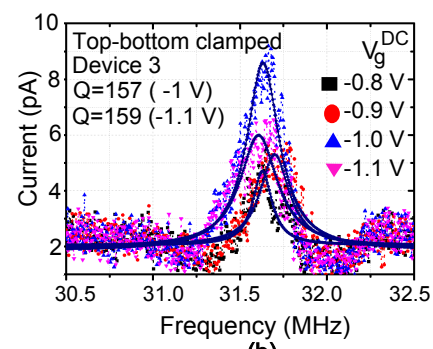

(b)

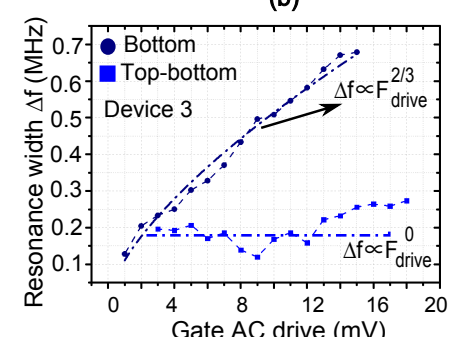

(d)
FIG. 4. Spectral response with a Lorentzian fit (measured with a setup similar to Figure S8-supplementary information) for (a) bottom clamped device and (b) top-bottom clamped device. Both devices were measured with biasing parameters as $V_{g}^{a c}=18 \mathrm{mV}, V_{d s}^{a c}=10 \mathrm{mV}$ and $V_{d s}^{D C}=10 \mathrm{mV}$. (c) The spectral responses from 5 individual measurements and $\mathrm{Q}$ factors were extracted from the Lorentzian fit and plotted for various $V_{g}^{D C}$. (d) Resonance width as a function of AC gate bias with a fixed DC gate bias $V_{g}^{D C}=1 V$ for two clamping configurations. The significant broadening effect with increasing gate drive is visible for the bottom clamped device.

1. A non-linear spectral response makes it difficult to distinguish between Duffing non-linearity and non-linear damping. Normalization of the resonance response to the drive force provides a simple and useful scheme to observe non-linear damping. The output resonance current (conduction modulation current based on field effect induced charge modulation-supplementary information section-4-Eq.2) follows $I \propto z V_{g}^{D C}$ where $z \propto \frac{F_{\text {drive }}}{k} Q$. Scaling the resonance response with a normalization factor $\left(V_{g}^{D C} / \omega_{0}\right)^{2}$ results in a scaled current $I_{\text {norm }} \propto Q$ (for simplicity, $\omega_{0}=\omega$ at $I_{\max }$ is used). The factor $\omega_{0}^{2}$ was used to account for the changing resonance frequency due to gate induced tension and changing spring constant $k\left(\propto \omega_{0}^{2}\right)$. With linear damping, the scaled peak currents would have the same height and in presence of non-linear damping, one would observe a decrease in the current with increasing drive force due to increased damping (lower Q). Such a normalization technique was analytically reported by Lifshitz et.al. ${ }^{29}$ and also by Imboden et.al. ${ }^{30}$ for non-linear damping investigation in diamond nanoresonators.

Figure 5 shows the normalized resonance response (with respect to driving force) for a bottom clamped (Device 2) and a top-bottom clamped resonator (Device 1). For the bottom clamped device, with increasing drive force i.e. higher DC gate bias, the response exhibits both 
spring hardening and a decrease in the peak currents, which is an indicative of non-linear damping. The topbottom clamped device exhibits no significant drop in peak currents while spring hardening is clearly visible.

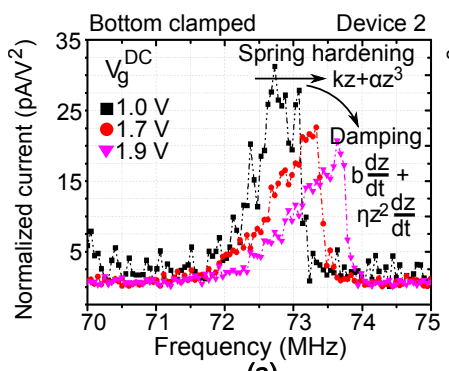

(a)

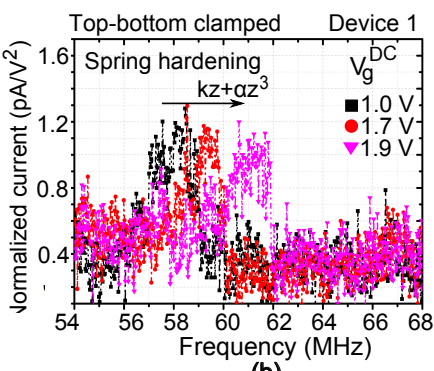

(b)
FIG. 5. (a) Normalized resonance spectral response for the bottom clamped Device 2 . The right frequency shift is attributed to the gate dependent spring hardening effect while the drop in the normalized resonance current with increasing DC gate bias is a result of increased damping; (b) Normalized resonance spectral response for the top-bottom clamped device with only an observable spring hardening effect and no significant change in the normalized resonance currents with increasing DC gate bias. Both devices were measured with biasing parameters $V_{g}^{a c}=15 \mathrm{mV}, V_{d s}^{a c}=$ $10 \mathrm{mV}$ and $V_{d s}^{D C}=10 \mathrm{mV}$.

\section{DISCUSSION AND CONCLUSION}

Non-linear damping so far has only been considered phenomenologically. Eichler et. al. ${ }^{26}$ argued that such dissipation mechanism stems from external channels such as clamping losses. Internal mechanisms such as the viscoelastic effect could also partially account for the non-linear dissipation behavior in nanoresonators as reported by Zaitsev et. al. ${ }^{27}$. Geometric nonlinearity might also be a root cause of spectral broadening but the experimental biases were kept below the onset of Duffing mode to avoid geometric non-linearities. Similar spectral broadening due to electrostatic measurement noise might also occur but the device with both clamping configurations was subjected to the same measurement noise. The investigation of energy dissipation using non-linear dynamics reported here suggest a possible association to external clamping mechanisms, in particular from device 3 where a same nanotube was subjected to two different clamping configurations.

As seen in Device 3 from Figure 4a,b the top-bottom clamped device resonates at $31 \mathrm{MHz}$, a further decrease of $9 \mathrm{MHz}$ after platinum ALD was observed. As such the device has undergone a change due to topmetallization and might limit our direct comparison of clamping effect and Q-factor improvement. Decrease in the nanotube tension or mass loading effect are two possible explanations for the observed change in the resonance frequency (supplementary informationsection 5). Assuming that the change in nanotube tension (or spring constant $k$ ) does not effect the energy dissipation (b), Q factors are expected to decrease due to decrease in the resonance frequency. Similarly for mass loading, increased surface losses would result in lower $\mathrm{Q}$ factors in contrast to the observed increase in Q-factors. The decrease in non-linear damping with the change in clamping configuration suggests that the energy dissipation occurs (or partially) through external clamping mechanisms. If one considers the energy lost per cycle at resonance, we obtain for the present scenario (supplementary information-section 6)

$$
\frac{1}{Q_{\text {Bottom }}}=\frac{1}{Q_{\text {Top-bottom }}}+\frac{\eta z^{2}}{8 \pi m f_{0}}
$$

For a Q-factor change of two fold, amplitude dependent damping in bottom clamped device results from a nanotube's vibrational displacement $\mathrm{z}=11 \mathrm{~nm}$ (nanotube suspended length of $2 \mu \mathrm{m}$ ) and increases with increasing nanotube displacement.

While the improved Q-factors after top-metallization are still not exceptionally high, other hidden mechanisms such as frequency fluctuations ${ }^{31}$ or $1 / \mathrm{f}^{\text {noise }}{ }^{9}$ might limit the extracted Q-factors. Viscoelastic effect was also recently reported in nanotube based cantilevers at room temperature to be a significant contributor to nonlinear damping ${ }^{32}$. Viscoelastic model ${ }^{27}$ which predicts $\eta=4 b / r^{2}$ ( $\mathrm{r}$ is the radius of the nanotube), results in $\eta=13000 \mathrm{~kg} \mathrm{~m} \mathrm{~m}^{-2} \mathrm{~s}^{-1}$ which is two orders of magnitude larger than the observation. Such a large value together with clamping losses can significantly limit the room temperature quality factor in carbon nanotube beam resonators. In contrast to one-dimensional clamping in nanotube resonator, two-dimensional clamping such as in graphene resonators also exhibited improved quality factors with additional clamping ${ }^{33}$. The improvement was attributed to the suppression of free edges with additional clamping metal leading to lower energy dissipation.

Apart from energy dissipation, the weak clamping behavior reported here argues against perfect clamping conditions which are often used in various theoretical and experimental work. These weak surface interactions might affect energy dissipation such as breaking and healing of surface bonds during motion (velcro effect) and can be enhanced at higher motional amplitude ${ }^{34}$. Such a mechanism might be feasible considering the onset of slipping to occur at $\sim 7.4 \mathrm{~nm}$ (in comparison to $\sim 11 \mathrm{~nm}$ extracted for non-linear amplitude dependent damping). The existence of nanotube tension (47 pN $150 \mathrm{pN}$ ) due to clamping also suggest a significant role of external mechanisms such as van-der-Waals adhesive forces (or surface/edge and frictional interactions) on the dynamic behavior of nanoresonators. Such a scenario has been theoretically studied for carbon nanotubes ${ }^{35}$ where 
surface van-der- Waals forces can induce substantial axial and radial deformation leading to a build up of nanotube tension. Son et. al. ${ }^{36}$ also investigated and modeled the axial tension on suspended SWCNTs due to surface interactions at clamping sites predicting radial or axial forces in the order of $10 \mathrm{pN} / \mathrm{nm}$. The resonance frequency downshift and saturation shown in Figure 2 provides a way to demonstrate experimentally the impact of clamping and complements the theoretical work in nanoresonators. These surface interactions could be modified and clamping strength as well as energy dissipation can arguably be also improved by increasing the thickness of top metal or alternatively using other materials

In summary, we investigated the effect of two different clamping configurations on nanotube based resonator devices. The nanotube attached to the underlying palladium electrodes resulted in a bottom clamped device and later, with selective top metallization on the electrodes, resulted in a top-bottom clamped device. In contrast to the perfect clamping assumptions, slippage and weak clamping behavior were observed as a resonance frequency downshift and was demonstrated with both clamping configurations. Significant reduction of amplitude dependent non-linear damping in topbottom clamped devices was observed. A direct comparison on the same nanotube device subjected to both clamping configuration allowed us to quantify the non-linear and linear damping parameters due to clamping effects and also suggest that the energy dissipation path linked to non-linear damping stems partially in external clamping mechanisms and gives us further useful insight on the impact of clamping in nanoresonators.

\section{SUPPLEMENTARY MATERIAL}

Additional details about DC transfer characteristic measurements, Raman spectroscopy, lock-in detection technique for RF characterization, measurement history cycle and additional measurements for all devices, theoretical calculations and analytical model can be found in the supplementary information.

\section{ACKNOWLEDGMENTS}

The authors thank Sebastian Eberle for support in nanotube growth, Raphael Steffen for useful discussion, clean room facility Binnig and Rohrer Nanotechnology Center (BRNC) at IBM Research, Zurich and FIRST CLA at ETH Zurich for equipment accessibility and useful discussion. We would also like to kindly acknowledge the Swiss National Science Foundation (SNSF-Project No. 153292) for the funding support.
${ }^{1}$ H. B. Peng, C. W. Chang, S. Aloni, T. D. Yuzvinsky, and A. Zettl, "Ultrahigh frequency nanotube resonators," Physical Review Letters 97, 2-5 (2006).

${ }^{2}$ K. Jensen, K. Kim, and A. Zettl, "An atomic-resolution nanomechanical mass sensor," Nature Nanotechnology 3, 533537 (2008).

${ }^{3} \mathrm{M}$. Imboden and P. Mohanty, "Dissipation in nanoelectromechanical systems," Physics Reports 534, 89146 (2014).

${ }^{4}$ K. Kunal and N. R. Aluru, "Akhiezer damping in nanostructures," Physical Review B 84, 245450 (2011).

${ }^{5}$ R. Lifshitz and M. L. Roukes, "Thermoelastic damping in microand nanomechanical systems," Physical Review B 61, 5600-5609 (2000).

${ }^{6}$ L. Villanueva and S. Schmid, "Evidence of Surface Loss as Ubiquitous Limiting Damping Mechanism in SiN Micro- and Nanomechanical Resonators," Physical Review Letters 113, 227201 (2014).

${ }^{7}$ J. A. Judge, D. M. Photiadis, J. F. Vignola, B. H. Houston, and J. Jarzynski, "Attachment loss of micromechanical and nanomechanical resonators in the limits of thick and thin support structures," Journal of Applied Physics 101, 013521 (2007).

${ }^{8}$ J. Lee, Z. Wang, K. He, J. Shan, and P. X.-L. Feng, "Air damping of atomically thin MoS2 nanomechanical resonators," Applied Physics Letters 105, 023104 (2014).

${ }^{9}$ J. Moser, A. Eichler, J. Güttinger, M. I. Dykman, and A. Bachtold, "Nanotube mechanical resonators with quality factors of up to 5 million," Nature Nanotechnology 9, 1007-1011 (2014).

${ }^{10}$ H. Jiang, M.-F. Yu, B. Liu, and Y. Huang, "Intrinsic Energy Loss Mechanisms in a Cantilevered Carbon Nanotube Beam Oscillator," Physical Review Letters 93, 185501 (2004).

${ }^{11}$ X. Liu, J. F. Vignola, H. J. Simpson, B. R. Lemon, B. H. Houston, and D. M. Photiadis, "A loss mechanism study of a very high Q silicon micromechanical oscillator," Journal of Applied Physics 97, 023524 (2005).

${ }^{12}$ B. Zhang, L. Zhao, Y. Cheng, D. Golberg, and M.-S. Wang, "Reversible Tuning of Individual Carbon Nanotube Mechanical Properties via Defect Engineering," Nano Letters 16, 5221-5227 (2016).

${ }^{13}$ V. Sazonova, Y. Yaish, H. Üstünel, D. Roundy, T. A. Arias, and P. L. McEuen, "A tunable carbon nanotube electromechanical oscillator," Nature 431, 284-287 (2004).

${ }^{14}$ J. H. Ko, J. Jeong, J. Choi, and M. Cho, "Quality factor in clamping loss of nanocantilever resonators," Applied Physics Letters 98, 171909 (2011).

${ }^{15}$ K. Jensen, H. Peng, and A. Zettl, "Limits of Nanomechanical Resonators," 2006 International Conference on Nanoscience and Nanotechnology, Proceedings of the IEEE International Conference on Nanoscience and Nanotechnology , 68-71 (2006).

${ }^{16}$ D. M. Photiadis and J. A. Judge, "Attachment losses of high Q oscillators," Applied Physics Letters 85, 482-484 (2004).

${ }^{17}$ Z. Hao, A. Erbil, and F. Ayazi, "An analytical model for support loss in micromachined beam resonators with in-plane flexural vibrations," Sensors and Actuators A: Physical 109, 156-164 (2003).

${ }^{18}$ D. S. Bindel and S. Govindjee, "Elastic PMLs for resonator anchor loss simulation," International Journal for Numerical Methods in Engineering 64, 789-818 (2005).

${ }^{19}$ M. Aykol, B. Hou, R. Dhall, S. W. Chang, W. Branham, J. Qiu, and S. B. Cronin, "Clamping Instability and van der Waals Forces in Carbon Nanotube Mechanical Resonators," Nano Letters 14, 2426-2430 (2014).

${ }^{20}$ L. Kumar, L. V. Jenni, M. Haluska, C. Roman, and C. Hierold, "Mechanical stress relaxation in adhesively clamped carbon nanotube resonators," AIP Advances 8, 025118 (2018).

${ }^{21}$ X. Huang, M. Prakash, C. Zorman, M. Mehregany, and M. Roukers, "Free-free beam silicon carbide nanomechanical resonators," Transducers 2003, 12th International Conference on Solid-State Sensors, Actuators and Microsystems, 
Proceedings of Transducers 2003, 12th International Conference on Solid-State Sensors, Actuators and Microsystems 1, 342-343 (2003).

22 J. Rieger, A. Isacsson, M. J. Seitner, J. P. Kotthaus, and E. M. Weig, "Energy losses of nanomechanical resonators induced by atomic force microscopy-controlled mechanical impedance mismatching," Nature Communications 5, 3345 (2014).

${ }^{23}$ L. V. Jenni, M. Haluska, and C. Hierold, "Selective metallization and passivation of dry-transferred carbon nanotubes in fieldeffect transistors," 2018 IEEE Micro Electro Mechanical Systems (MEMS), Proceedings of the IEEE International Conference on Micro Electro Mechanical Systems (MEMS) , 479-482 (2018).

${ }^{24} \mathrm{M}$. Muoth and C. Hierold, "Transfer of carbon nanotubes onto microactuators for hysteresis-free transistors at low thermal budget," Proceedings of the IEEE International Conference on Micro Electro Mechanical Systems (MEMS) , 1352-1355 (2012).

${ }^{25}$ A. J. M. Mackus, M. A. Verheijen, N. Leick, A. A. Bol, and W. M. M. Kessels, "Influence of Oxygen Exposure on the Nucleation of Platinum Atomic Layer Deposition: Consequences for Film Growth, Nanopatterning, and Nanoparticle Synthesis," Chemistry of Materials 25, 1905-1911 (2013).

${ }^{26}$ A. Eichler, J. Moser, J. Chaste, M. Zdrojek, I. Wilson-Rae, and A. Bachtold, "Nonlinear damping in mechanical resonators made from carbon nanotubes and graphene," Nature Nanotechnology 6, 339-342 (2011).

${ }^{27}$ S. Zaitsev, O. Shtempluck, E. Buks, and O. Gottlieb, "Nonlinear damping in a micromechanical oscillator," Nonlinear Dynamics 67, 859 (2012).
${ }^{28}$ L. Kumar, L. V. Jenni, M. Haluska, C. Roman, and C. Hierold, "Clamping and Q-factor Improvement in a Carbon Nanotube Resonator," Proceedings of Eurosensors 2 (13), 848 (2018).

${ }^{29}$ R. Lifshitz and M. Cross, "Nonlinear Dynamics of Nanomechanical Resonators," in Nonlinear Dynamics of Nanosystems (Wiley-VCH, 2010) pp. 221-266.

${ }^{30} \mathrm{M}$. Imboden, O. Williams, and P. Mohanty, "Nonlinear dissipation in diamond nanoelectromechanical resonators," Applied Physics Letters 102, 103502 (2013).

${ }^{31}$ M. Sansa, E. Sage, E. C. Bullard, M. Gély, T. Alava, E. Colinet, A. K. Naik, L. G. Villanueva, L. Duraffourg, M. L. Roukes, et al., "Frequency fluctuations in silicon nanoresonators," Nature nanotechnology 11, 552 (2016).

${ }^{32}$ A. Descombin, P. Poncharal, A. Pascale-Hamri, M. Choueib, R. Diehl, P. Vincent, S. T. Purcell, a. ayari, and S.-M. Perisanu, "Giant, voltage tuned, q-factors of single wall carbon nanotubes and graphene at room temperature," Nano letters 19, 1534-1538 (2019).

${ }^{33}$ M. Takamura, H. Okamoto, K. Furukawa, H. Yamaguchi, and H. Hibino, "Energy Dissipation in Graphene Mechanical Resonators with and without Free Edges," Micromachines 7, 158 (2016).

${ }^{34}$ C. Seoanez, F. Guinea, and A. C. Neto, "Dissipation in graphene and nanotube resonators," Physical Review B 76, 125427 (2007).

${ }^{35}$ T. Hertel, R. E. Walkup, and P. Avouris, "Deformation of carbon nanotubes by surface van der Waals forces," Physical Review B 58, 125427 (1998).

${ }^{36}$ H. Son, G. G. Samsonidze, J. Kong, Y. Zhang, X. Duan, J. Zhang, Z. Liu, and M. S. Dresselhaus, "Strain and friction induced by van der Waals interaction in individual single walled carbon nanotubes," Applied Physics Letters 90, 253113 (2007). 